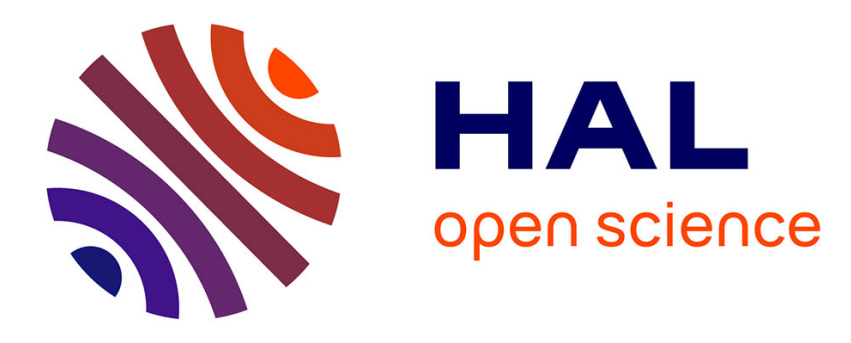

\title{
Model for thermal convection with uniform volumetric energy sources \\ Mathieu Creyssels
}

\section{To cite this version:}

Mathieu Creyssels. Model for thermal convection with uniform volumetric energy sources. Journal of Fluid Mechanics, 2021, 919, 10.1017/jfm.2021.392 . hal-03033712v2

HAL Id: hal-03033712

https://hal.science/hal-03033712v2

Submitted on 28 Apr 2021

HAL is a multi-disciplinary open access archive for the deposit and dissemination of scientific research documents, whether they are published or not. The documents may come from teaching and research institutions in France or abroad, or from public or private research centers.
L'archive ouverte pluridisciplinaire HAL, est destinée au dépôt et à la diffusion de documents scientifiques de niveau recherche, publiés ou non, émanant des établissements d'enseignement et de recherche français ou étrangers, des laboratoires publics ou privés. 


\title{
Model for thermal convection with uniform volumetric energy sources
}

\author{
M. Creyssels $\dagger$ \\ Ecole Centrale de Lyon, CNRS, Univ Claude Bernard Lyon 1, INSA Lyon, LMFA, UMR5509, \\ 69130, Ecully, France
}

(Received xx; revised xx; accepted xx)

A theoretical model is derived to predict both the heat fluxes at the upper and lower horizontal surfaces of an internally heated $(\mathrm{IH})$ convection cell by extending the wellknown Grossmann \& Lohse (2001) theory. The approach of Creyssels (2020) is generalized for a fluid heated internally and uniformly, confined between top and bottom plates of equal temperature. For each plate, a Nusselt number is defined and an analytical formula is given to predict its variations with the Rayleigh and Prandtl numbers. The turbulent flow produced in the upper half of the IH convection cell is very similar to that observed in standard Rayleigh-Bénard (RB) convection. On the contrary, the lower plate is swept by the large scale flow that circulates through the entire cell. The corresponding boundary layer is therefore modelled by a laminar boundary layer of the Blasius type. These predictions are consistent with the independent theoretical scalings proposed by Wang et al. (2021) and they are confirmed by the numerical results obtained by Goluskin \& van der Poel (2016); Wang et al. (2021).

\section{Introduction}

Natural convection induced by internal heat production is a phenomenon that occurs very often in geophysical, astrophysical and engineering systems (Goluskin 2015). By following the analysis recently given by Creyssels (2020), the purpose of this paper is to extend the many theoretical, numerical and experimental results of Rayleigh-Bénard (RB) convection to two internally heated (IH) convection systems shown in Figs. 1 (a) and (b). In RB convection, convective flow is produced by thermal boundary conditions that cause heat to enter through the lower hot plate and exit through the upper cold plate. In this case, convection is controlled by the temperature difference between the plates $(\Delta T)$ and the height of the cell $(h)$, or by the Rayleigh number defined as

$$
R a=\frac{g \beta \Delta T h^{3}}{\nu \kappa}
$$

where $g$ is the uniform gravitational acceleration, $\beta, \nu$ and $\kappa$ are, respectively, the coefficient of thermal expansion, the kinematic viscosity and the thermal diffusivity of the fluid. On the contrary, for IH convection, the flow is produced by a volumetric source of internal heating $\left(q\right.$ in $\left.\mathrm{W} / \mathrm{m}^{3}\right)$, itself produced by chemical or nuclear reactions, or by radiation. Instead of using $R a$, the Rayleigh-Roberts number (Roberts 1967) is adopted as follows

$$
R r=\frac{g \beta q h^{5}}{\lambda \nu \kappa}
$$

where $\lambda$ is the thermal conductivity of the fluid. A first approach of $\mathrm{IH}$ convection is to take $q$ constant and uniform throughout the volume. Using $h^{2} / \kappa$ as the unit of time, $h$

$\dagger$ Email address for correspondence: mathieu.creyssels@ec-lyon.fr 


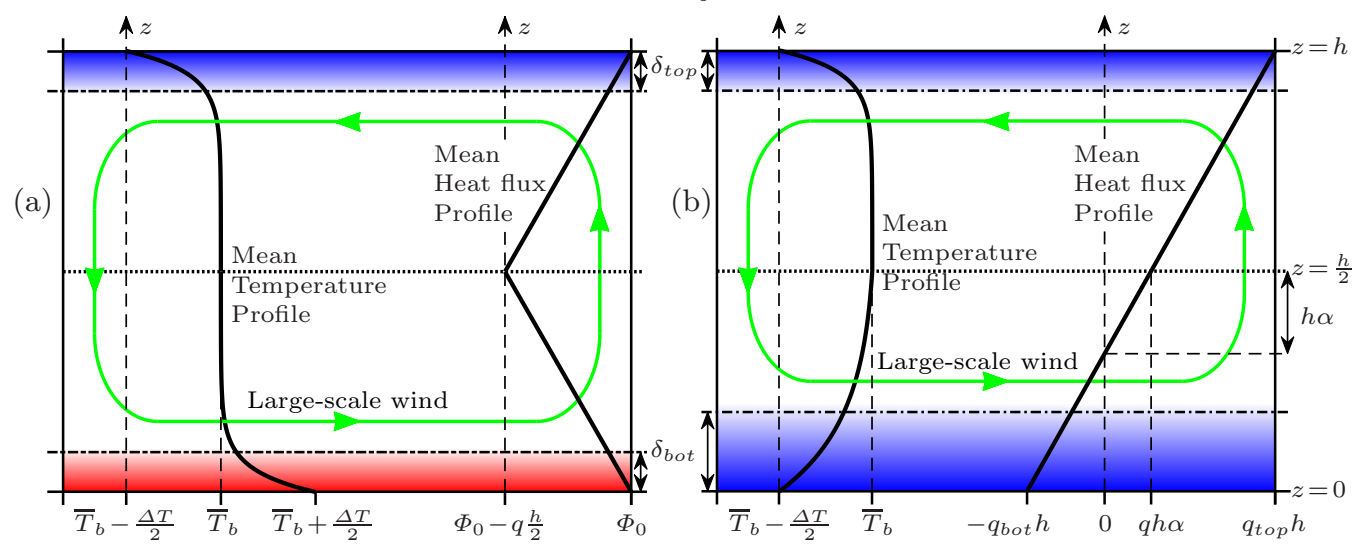

Figure 1. Two convective systems are theoretically studied here. Configuration (a) is closer to standard RB experiments since the two hot and cold plates play the same role and the same heat flux $\left(\Phi_{0}\right)$ crosses them. But the heat flux at the center of the cell is lower or greater than $\Phi_{0}$, depending on the sign of $q: \bar{\Phi}_{a}(z=h / 2)=\Phi_{0}-q h / 2$. Configuration (b) is more complex because the upper half cell is very similar to the upper half cell of RB experiments whereas the flow is stratified in the lower part of the bottom half cell. The heat flux at the bottom plate is negative $\left(-q_{b o t} h\right)$ and is not equal in absolute value to the heat flux at the top plate $\left(q_{t o p} h\right)$. Besides, $q_{t o p}>q_{\text {bot }}$. For configuration (b), the mean temperature profile $\bar{T}(z)$ was measured by Goluskin \& van der Poel (2016). The upper and lower thermal boundary layers of thickness $\delta_{\text {top }}$ and $\delta_{b o t}$ are also displayed for each configuration.

as the unit of length and $q h^{2} / \lambda$ as the unit of temperature, the dimensionless Boussinesq equations governing the velocity, pressure and temperature are the incompressibility condition $(\boldsymbol{\nabla} \cdot \boldsymbol{u})$ and

$$
\begin{aligned}
& \tilde{\partial}_{t} \tilde{\boldsymbol{u}}+\tilde{\boldsymbol{u}} \cdot \tilde{\nabla} \tilde{\boldsymbol{u}}=-\tilde{\nabla} \tilde{p}+\operatorname{Pr} \tilde{\nabla}^{2} \tilde{\boldsymbol{u}}+\operatorname{Pr} \operatorname{Rr} T \hat{\boldsymbol{e}}_{z}, \\
& \tilde{\partial}_{t} \tilde{T}+\tilde{\boldsymbol{u}} \cdot \tilde{\nabla} \tilde{T}=\tilde{\nabla}^{2} \tilde{T}+1
\end{aligned}
$$

where dimensionless variables and operators are designated by tildes, $\hat{\boldsymbol{e}}_{z}$ is the vertical unit vector and $P r$ is the Prandtl number. With regard to thermal boundary conditions, we consider hereafter those used by most of previous studies i.e. fixed and equal temperature conditions $\left(T_{0}\right)$ at the top and bottom plates. By adopting this condition, modeling an IH convection experiment becomes a great challenge because in a single cell, there are both positive and negative vertical mean temperature gradients (see Fig. 1b). This leads to both turbulent convection and a stably stratified lower boundary layer. Therefore, the experiments on RB and IH convection have some similarities but also some differences. For both cases, the mean temperature is almost constant in the middle of the cell and we call it the temperature of the 'bulk flow' $\left(\bar{T}_{b}\right)$. At high $R a$ numbers and assuming negligible non-Boussinesq effects, $\bar{T}_{b}$ is equal to the average of the top and bottom plate temperatures for a $\mathrm{RB}$ experiment, while $\bar{T}_{b}$ is the maximum of the mean temperature in an IH convection cell (see Fig. 1b). Besides, the two thermal boundary layers located near the top and bottom plates have the same thickness for a RB experiment. On the contrary, heating in volume leads to an asymmetry between the two thermal boundary layers. The mean temperature profile was measured by Goluskin \& van der Poel (2016) and is shown in Fig. 1 (b). Therefore, the upper boundary layer is similar to that observed in a RB experiment, whereas in the lower boundary layer, the mean temperature gradient tends to stop convective flows produced in the upper region of the cell. Consequently, in an IH convection cell, the thickness of the lower boundary layer is greater than that 


$\begin{array}{ccccc}\text { Reference } & P r & R r \text { range } & N u_{\text {top }} \text { fit } & N u_{\text {bot }} \text { fit } \\ \text { Laboratory experiments } & & & & \\ \text { Kulacki \& Goldstein }(1972) & \approx 6 & 5 \cdot 10^{4}-2 \cdot 10^{7} & 0.18 R r^{0.23} & 0.66 R r^{0.10} \\ \text { Jahn \& Reineke }(1974) & \approx 7 & 10^{5}-10^{9} & 0.39 R r^{0.20} & 1.07 R r^{0.10} \\ \text { Mayinger et al. }(1975) & \approx 7 & 10^{5}-10^{11} & 0.22 R r^{0.23} & 0.68 R r^{0.10} \\ \quad \text { Ralph et al. }(1977) & \approx 7 & 4 \cdot 10^{8}-10^{12} & 0.10 R r^{0.24} & 0.14 R r^{0.17} \\ \quad \text { Lee } \text { et al. (2007) } & \approx 0.7 & 10^{10}-4 \cdot 10^{11} & 0.09 R r^{0.24} & 0.71 R r^{0.11} \\ \text { Simulations (3D DNS) } & & & & \\ \text { Wörner et al. } \text { (1997) } & 7 & 10^{5}-10^{8} & 0.19 R r^{0.23} & 0.65 R r^{0.10} \\ \text { Goluskin \& van der Poel (2016) } & 1 & 5 \cdot 10^{7}-2 \cdot 10^{10} & 0.18 R r^{0.23} & 0.38 R r^{0.14}\end{array}$

TABle 1. Previous results giving Nusselt numbers as a function of $R r$ for experiments and numerical simulations of three-dimensional IH convection (Goluskin 2015).

of the upper boundary layer $\left(\delta_{b o t}>\delta_{t o p}\right)$. In addition, the difference in thickness must increase as the Rayleigh number increases. Likewise, at a fixed $R a$ number, the mean vertical heat flux is constant for a RB experiment and is given by the Nusselt number as $\Phi_{R B}=N u_{R B} \frac{\lambda \Delta T}{h}$. In contrast, in an IH convection cell, the heat produced inside the fluid is evacuated through both lower and upper boundaries, leading to a mean vertical heat flux that changes sign from the bottom plate to the top plate (see Fig. 1b). As the mechanisms that drive the two thermal boundary layers are different, the heat fluxes through the upper and lower plates are not equal in absolute value. A coefficient $\alpha$ can be defined to quantify this down-up asymmetry. Indeed, the fraction of heat produced inside the fluid flowing outwards from the bottom plate can be written as

$$
q_{b o t}=\left(\frac{1}{2}-\alpha\right) q
$$

leading to a heat flux at the bottom plate equal to $-q_{b o t} h \vec{e}_{z}$. In steady state, energy conservation yields to a heat flux that leaves through the top plate as $q_{t o p} h \vec{e}_{z}$, with

$$
q_{\text {top }}=\left(\frac{1}{2}+\alpha\right) q
$$

Then, for each plate, we can define one Nusselt number as

$$
\begin{aligned}
& N u_{\text {bot }}=\frac{q_{b o t} h^{2}}{\lambda \Delta T}, \\
& N u_{\text {top }}=\frac{q_{t o p} h^{2}}{\lambda \Delta T} .
\end{aligned}
$$

To be consistent with the definition of the Nusselt number adopted for RB convection experiments, the characteristic temperature difference $\Delta T$ used in (1.7) and (1.8) is: $\Delta T=2\left(\bar{T}_{b}-T_{0}\right)$. There is therefore a factor 2 by comparing the definitions of $N u_{b o t}$ and $N u_{\text {top }}$ given in previous studies on IH convection (Goluskin 2015). Note also that other definitions of Nusselt numbers are given in the literature. Instead of using $\bar{T}_{b}$, the mean fluid temperature $(\langle\bar{T}\rangle$, where angle brackets denote an average over the entire volume) can be chosen as temperature reference in (1.7) and (1.8). Figure 6 (a) shows that the difference of temperature $\bar{T}_{b}-\langle\bar{T}\rangle$ becomes negligible only for very high Rayleigh-Roberts numbers.

Kulacki \& Goldstein (1972); Jahn \& Reineke (1974); Mayinger et al. (1975); Ralph et al. (1977); Lee et al. (2007); Wörner et al. (1997); Goluskin \& van der Poel (2016) have measured experimentally and numerically the Nusselt numbers $N u_{\text {top }}$ and $N u_{\text {bot }}$ as 
a function of the control parameter $R r$. As is usual for RB experiments, they presented their results as power-law fits. $N u_{t o p}$ has been found to increase with $R r$ at rates between $R r^{0.20}$ and $R r^{0.24}$ (see Table 1 ). $N u_{\text {bot }}$ increases more slowly with $R r$, at rates between $R r^{0.10}$ and $R r^{0.17}$. Up to now, no theory has been able to predict these exponents whereas for RB convection, the theory developed by Grossmann \& Lohse (2000); Grossmann \& Lohse (2001) (henceforth the GL theory, Eqs. (2.18) and (2.19)) describes well the behaviour of $N u_{R B}(R a, P r)$ and $R e_{R B}(R a, P r)$.

Creyssels (2020) has recently developed a simple theoretical model to predict the $R a$ and Pr-dependent Nusselt number for a modified RB experiment in which heat is injected by volume but only in the lower thermal boundary layer. At the same time, the upper boundary layer is cooled with the same rate in order to have a constant energy in the convection cell, as in a standard RB cell. Note that Lepot et al. (2018); Bouillaut et al. (2019) presented an experimental method to bypass the cooling boundary layer in order to perform this modified RB experiment. For configuration shown in Fig. 1 (b), the problem is much more complex since the symmetry between the two plates is broken. Because of this, GL theory is first extended to the modified RB configuration (a) (see Fig. 1a) for which the fluid is confined between two horizontal plates of different temperature ( $\Delta T>0$ is fixed). To preserve the symmetry between the two plates, the lower part of the cell for configuration (a) is cooled with the same volumetric power as the upper part is heated:

$$
q_{v}(z)= \begin{cases}-q, & \text { for } 0 \leqslant z \leqslant h / 2, \\ +q, & \text { for } h / 2 \leqslant z \leqslant h .\end{cases}
$$

In steady state, energy conservation yields to the following variation of the heat flux averaged over a horizontal cross-section:

$$
\bar{\Phi}_{a}(z)=\left\{\begin{array}{l}
\Phi_{0}-q z, \quad \text { for } 0 \leqslant z \leqslant h / 2, \\
\Phi_{0}-q(h-z), \quad \text { for } h / 2 \leqslant z \leqslant h,
\end{array}\right.
$$

where $\Phi_{0}$ is the heat flux that crosses each horizontal plate. For configuration (a), the Nusselt number can then be defined as for standard RB experiment using

$$
N u_{a}=\frac{\Phi_{0} h}{\lambda \Delta T} .
$$

The Nusselt $\left(N u_{a}\right)$ and Reynolds $\left(R e_{a}\right)$ numbers depend on three non-dimensional parameters: $R a, \operatorname{Pr}$ and $Q$, where $Q$ represents the non-dimensional form of the volumetric heating or cooling sources:

$$
Q=\frac{q h^{2}}{\lambda \Delta T}
$$

Note that: (i) $N u_{a}=N u_{R B}(R a, P r)$ when $Q=0$; (ii) $Q$ can be either positive or negative; (iii) a priori, $N u_{a}<N u_{R B}$ and $R e_{a}<R e_{R B}$ when $Q>0$ whereas $N u_{a}>N u_{R B}$ and $R e_{a}>R e_{R B}$ if $Q<0$; (iv) $\bar{\Phi}_{a}(z)$ must be positive to avoid the weakening of the turbulent flow in the center of the cell and the appearance of a stratified flow. The condition $\bar{\Phi}_{a}(h / 2)=\Phi_{0}-q \frac{h}{2}>0$ implies that $Q<2 N u_{a}$. Finally, for $Q>0$, the upper boundary layer and the turbulent flow observed in the upper half of the cell of configuration (a) are similar to those observed in the upper half of a cell of configuration (b). However, $Q$ is a control parameter in configuration (a) while $Q$ must be measured or predicted in configuration (b) because $\Delta T=2\left(\bar{T}_{b}-T_{0}\right)$ is a function of $R r$ and $P r$. For configuration (b), using the Grossmann \& Lohse (2000) ansatz based on the decomposition of the kinetic and thermal dissipation rates, Wang et al. (2021) have theoretically modelled the inverse of $Q(\tilde{\Delta} \approx 1 / Q)$ by power-laws of 
$R r$ and $\operatorname{Pr}$ (see table 1 in Wang et al. (2021). In section 2, the GL theory is adapted to configuration (a) and the two equations (2.20) and (2.21) predict the evolution of $N u_{a}$ and $R e_{a}$ as a function of $R a, P r$ and $Q$. Under the same conditions (same $R a, P r$ and $Q$ ), we assume in sub-section 3.1 that both $N u_{\text {top }}$ and $R e_{b}$ measured in configuration (b) are given by $N u_{a}(R a, P r, Q)$ and $\operatorname{Re}_{a}(R a, P r, Q)$ (Eqs. (3.1) and (3.2)). In subsection 3.2, a theoretical model based on Prandtl-Blasius-Pohlhausen theory is derived to predict $N u_{b o t}$ as a function of the Reynolds number (Eq. (3.10)). Finally, these predictions for the $R a$ and $\operatorname{Pr}$ dependence of $N u_{t o p}$ and $N u_{b o t}$ are compared with threedimensional experimental and numerical results in section 4. A detailed comparison with the two-dimensional results of Goluskin \& Spiegel (2012); Wang et al. (2021) is given in Appendix A.

\section{Grossmann \& Lohse (2001) theory for RB experiment with volumetric energy sources (configuration a)}

To predict the variations of Nusselt numbers with $R a$ and $\operatorname{Pr}$ for internal heating and cooling convection experiments, Creyssels (2020) proposed to assume that, for high $R a$ numbers, the dynamical structure of the convective flow is the same as in standard RB experiments. Notably, he assumed that the 5 dimensionless parameters $\left(a, c_{1}-c_{4}\right)$ defined within the framework of the GL theory do not depend on the way the heat is injected and extracted in the experiment. Besides, the thickness of each thermal boundary layer is assumed to be only controlled by the difference of temperature between the bulk flow and the corresponding plate using the following equation [Eq. (2.1) in Creyssels (2020)]:

$$
\frac{\delta_{T}}{h}=\frac{1}{2 N u_{R B}(R a, P r)} .
$$

Then, the central idea of the GL theory is to split both mean kinetic energy and thermal dissipation rates into two contributions each, one from the bulk $(\mathrm{Bu})$ and one from the boundary layers (BLs) as

$$
\begin{aligned}
\left\langle\epsilon_{u}\right\rangle & =\left\langle\epsilon_{u}\right\rangle_{B u}+\left\langle\epsilon_{u}\right\rangle_{B L}, \\
\left\langle\epsilon_{T}\right\rangle & =\left\langle\epsilon_{T}\right\rangle_{B u}+\left\langle\epsilon_{T}\right\rangle_{B L},
\end{aligned}
$$

and each contribution is modelled as follows

$$
\begin{aligned}
\left\langle\epsilon_{u}\right\rangle_{B u} & \sim \frac{U_{a}^{2}}{h / U_{0}}\left(1-\frac{\delta_{u}}{h}\right) \approx \frac{\nu^{3}}{h^{4}} R e_{0}^{3}, \\
\left\langle\epsilon_{T}\right\rangle_{B u} & \sim \frac{(\Delta T)^{2}}{h / U_{a}^{\text {edge }}}\left(1-\frac{\delta_{T}}{h}\right) \approx \kappa\left(\frac{\Delta T}{h}\right)^{2} \operatorname{Re} e_{a} \operatorname{Prf}\left(\frac{\delta_{u}}{\delta_{T}}\right), \\
\left\langle\epsilon_{u}\right\rangle_{B L} & \sim \nu\left(\frac{U_{a}}{\delta_{u}}\right)^{2} \frac{\delta_{u}}{h}=\frac{\nu^{3}}{h^{4}} \operatorname{Re}_{a}^{2} \frac{h}{\delta_{u}}, \\
\left\langle\epsilon_{T}\right\rangle_{B L} & \sim \kappa\left(\frac{\Delta T}{\delta_{T}}\right)^{2} \frac{\delta_{T}}{h}=\kappa\left(\frac{\Delta T}{h}\right)^{2} \frac{h}{\delta_{T}} .
\end{aligned}
$$

In (2.4)-(2.6), $U_{a}$ represents the characteristic velocity of the bulk flow. Grossmann \& Lohse (2001) introduced the function $0 \leqslant f \leqslant 1$ because the relevant velocity at the edge between thermal BL and the thermal bulk can be less than $U_{a}$, depending on the ratio: $\delta_{u} / \delta_{T}$. They gave $f(x)=\left(1+x^{n}\right)^{-1 / n}$, with $n=4$ as an example of function $f$. Besides, 
they assumed that the velocity BLs are Blasius-like, with a thickness of

$$
\frac{\delta_{u}}{h}=\frac{a}{\sqrt{R e_{a}}} .
$$

For the mean kinetic energy and thermal dissipation rates, the balances of the turbulent kinetic energy and of the thermal variance give the following two exact relations (Creyssels 2020):

$$
\begin{aligned}
& \left\langle\epsilon_{u}\right\rangle=\frac{g \beta}{h}\left[\int_{0}^{h} \frac{\bar{\Phi}_{a}(z)}{\rho c_{p}} \mathrm{~d} z-\frac{\lambda \Delta T}{\rho c_{p}}\right], \\
& \left\langle\epsilon_{T}\right\rangle=\frac{1}{h} \int_{0}^{h} \bar{T}(z) \frac{q_{v}(z)}{\rho c_{p}} \mathrm{~d} z+\frac{\Delta T \Phi_{0}}{\rho c_{p} h} .
\end{aligned}
$$

Using (1.10), (1.1), (1.12) and (1.11), (2.9) becomes

$$
\left\langle\epsilon_{u}\right\rangle=\frac{\nu^{3}}{h^{4}} \frac{\left(N u_{a}-1-\frac{Q}{4}\right) R a}{\operatorname{Pr}^{2}} .
$$

As the GL theory is based on Prandtl-Blasius-Pohlhausen laminar boundary layers (Grossmann \& Lohse 2000), the mean temperature can be written as (Creyssels 2020):

$$
2 \frac{\bar{T}(z)-T_{b}}{\Delta T}= \begin{cases}1-\Theta_{P}\left(\frac{z}{\delta_{T}}\right), & \text { for } z \leqslant h / 2, \\ \Theta_{P}\left(\frac{h-z}{\delta_{T}}\right)-1, & \text { for } h / 2 \leqslant z \leqslant h,\end{cases}
$$

with $\Theta_{P}$ the Pohlhausen temperature profile which is assumed to be independent of the Prandtl number. In particular, $\Theta_{P}(0)=0$ and $\Theta_{P}(\eta) \rightarrow 1$ when $\eta \gg 1$. Using (1.9), (2.12), (1.12) and (1.11), (2.10) then becomes:

$$
\left\langle\epsilon_{T}\right\rangle=\kappa\left(\frac{\Delta T}{h}\right)^{2}\left[N u_{a}-\lambda_{d} \frac{\delta_{T}}{h} Q\right],
$$

where $\lambda_{d}=\int_{0}^{\infty}\left[1-\Theta_{P}(\eta)\right] \mathrm{d} \eta$ denotes the displacement thickness of the mean temperature profile ( $\lambda_{d} \approx 0.57$ for a Blasius profile).

From decomposition of the two global dissipation rates (2.2) and (2.3), four regimes of convection can be defined depending on whether the bulk or the BLs contributions dominate the global dissipations. For regimes $I I$ and $I V$, the kinetic energy dissipation rate is dominated by its bulk contribution whereas, for regimes $I$ and $I I I,\left\langle\epsilon_{u}\right\rangle \sim\left\langle\epsilon_{u}\right\rangle_{B L}$. Combining (2.4) and (2.11), or (2.6) and (2.11), and using (2.8), we obtain

$$
\frac{\left(N u_{a}-1-\frac{Q}{4}\right) R a}{P r^{2}} \sim\left(R e_{a}\right)^{\theta_{i}},
$$

whereas, for standard RB convection, we have

$$
\frac{\left(N u_{R B}-1\right) R a}{\operatorname{Pr}^{2}} \sim\left(R e_{R B}\right)^{\theta_{i}}
$$

with $\theta_{I I}=\theta_{I V}=3$ and $\theta_{I}=\theta_{I I I}=5 / 2$.

Regimes $I I I$ and $I V$ are obtained for high $R a$ numbers for which thermal dissipation rate is dominated by its bulk contribution whereas, for regimes $I$ and $I I,\left\langle\epsilon_{T}\right\rangle \sim\left\langle\epsilon_{T}\right\rangle_{B L}$. For standard RB convection, Grossmann \& Lohse (2000) predicted

$$
\left(N u_{R B}\right)^{\phi_{i}} \sim R e_{R B} \operatorname{Prf}\left(\frac{2 a N u_{R B}}{\sqrt{R e_{R B}}}\right),
$$



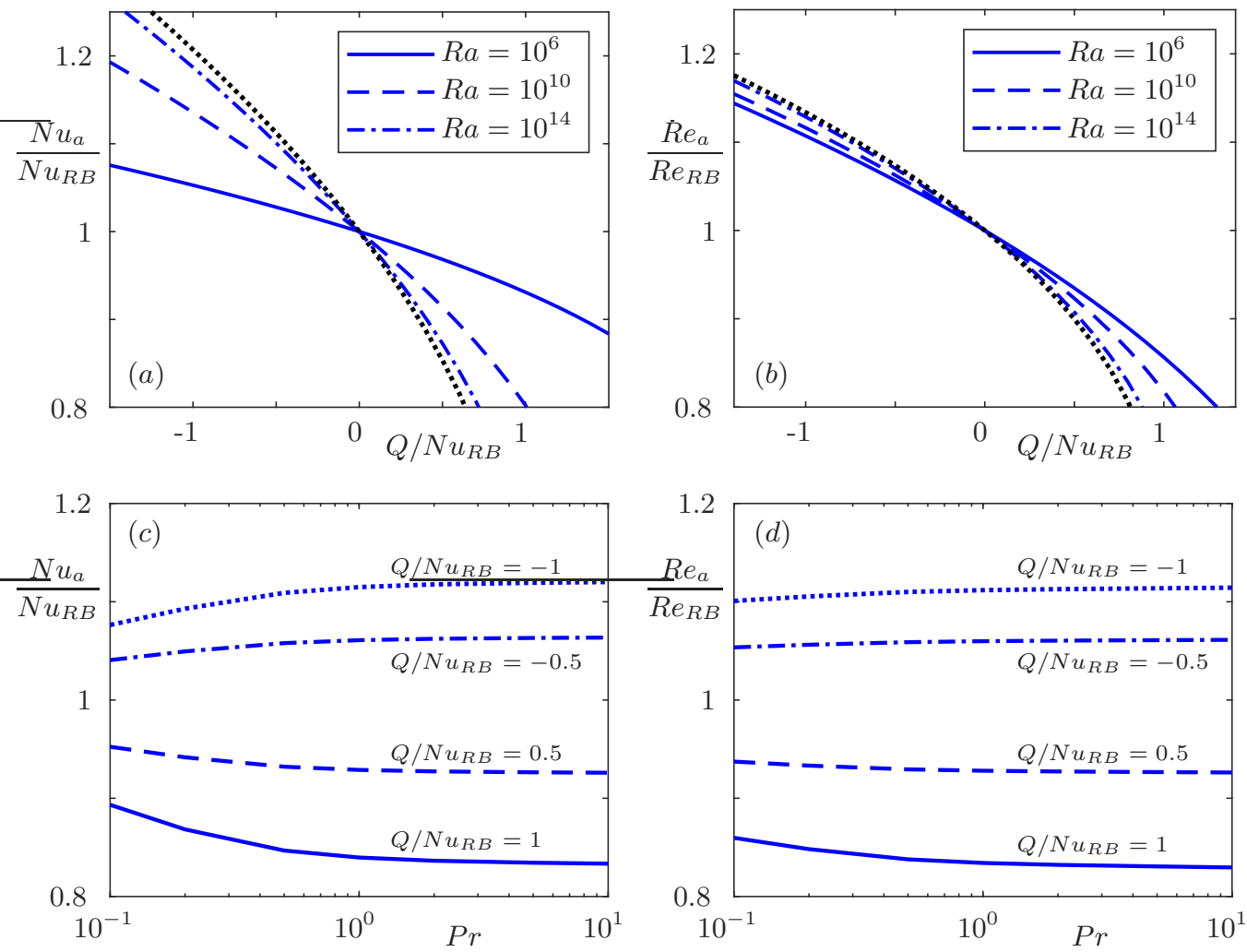

FiguRE 2. Results of the extension of GL theory for turbulent convection with uniform volumetric sources. $N u_{a} / N u_{R B}$ (a) and $R e_{a} / R e_{R B}$ (b) as a function of $Q$ normalized by $N u_{R B}(R a, P r)$ for $P r=1$ and for 3 different $R a$ numbers: $R a=10^{6}$ (solid line), $R a=10^{10}$ (dashed line) and $R a=10^{14}$ (dash-dotted line). The black dotted line represents regime $I V_{u}$ (Eq. 2.22) i.e. the limit of system of Eqs. (2.20) and (2.21) when $R a \rightarrow \infty$ and $\operatorname{Pr} \geqslant 1 . \quad N u_{a} / N u_{R B}$ (c) and $R e_{a} / R e_{R B}$ (d) as a function of $\operatorname{Pr}$ for $R a=10^{9}$ and from top to bottom: $Q / N u_{R B}=-1$, $-0.5,0.5$ and 1 .

with $\phi_{I I I}=\phi_{I V}=1$ and $\phi_{I}=\phi_{I I}=2$. For internal heating and cooling convection, combining (2.5) and (2.13), or (2.7) and (2.13), and using (2.1), it yields to

$$
N u_{a}-\frac{\lambda_{d}}{2} \frac{Q}{N u_{R B}} \sim\left\{\begin{array}{l}
\operatorname{Re} \operatorname{Prf}\left(\frac{2 a N u_{R B}}{\sqrt{R e_{a}}}\right) \quad(\text { regimes } I I I \text { and } I V), \\
\left.N u_{R B} \sim \sqrt{\operatorname{Re}_{R B} \operatorname{Prf}\left(\frac{2 a N u_{R B}}{\sqrt{R e_{R B}}}\right)} \quad \text { (regimes } I \text { and } I I\right) .
\end{array}\right.
$$

As the 4 previous regimes can only be observed experimentally and numerically for extreme values of $R a$ and $P r$ numbers, Grossmann \& Lohse (2001) proposed to describe $\mathrm{RB}$ convection at any $R a$ and $\operatorname{Pr}$ numbers as a mixture of these 4 regimes. Equations (2.15) and (2.16) are then generalized as

$$
\begin{aligned}
\left(N u_{R B}-1\right) \operatorname{RaPr}^{-2} & =\frac{c_{1}}{2 a} \operatorname{Re}_{R B}^{5 / 2}+c_{2} \operatorname{Re}_{R B}^{3}, \\
N u_{R B} & =c_{3} \sqrt{\operatorname{Re}_{R B} \operatorname{Prf}\left(\frac{2 a N u_{R B}}{\sqrt{R e_{R B}}}\right)}+c_{4} \operatorname{Re}_{R B} \operatorname{Prf}\left(\frac{2 a N u_{R B}}{\sqrt{R e_{R B}}}\right) .
\end{aligned}
$$


By applying this idea to internal heating convection, (2.14) and (2.17) are generalized using the two following equations

$$
\begin{aligned}
\left(N u_{a}-1-\frac{Q}{4}\right) \frac{R a}{\operatorname{Pr}^{2}} & =\frac{c_{1}}{2 a} R e_{a}^{5 / 2}+c_{2} R e_{a}^{3} \\
N u_{a}-\frac{\lambda_{d}}{2} \frac{Q}{N u_{R B}} & =c_{3} \sqrt{\operatorname{Re}_{R B} \operatorname{Prf}\left(\frac{2 a N u_{R B}}{\sqrt{R e_{R B}}}\right)}+c_{4} \operatorname{Re} e_{a} \operatorname{Prf}\left(\frac{2 a N u_{R B}}{\sqrt{R e_{a}}}\right) .
\end{aligned}
$$

Equations (2.20) and (2.21) give $N u_{a}$ and $R e_{a}$ numbers as functions of the 3 parameters $R a, \operatorname{Pr}$ and $Q$. Figures 2(a) and 2(b) show the ratios $N u_{a} / N u_{R B}$ and $R e_{a} / R e_{R B}$ as a function of $Q$ compensated by $N u_{R B}(R a, P r)$ for $\operatorname{Pr}=1$ and for 3 different $R a$ numbers: $R a=10^{6}$ (solid line), $R a=10^{10}$ (dashed line) and $R a=10^{14}$ (dash-dotted line). As expected, heating the lower part of the cell $(Q<0)$ increases both $N u_{a}$ and $R e_{a}$ while heating the upper part of the cell $(Q>0)$ decreases both $N u_{a}$ and $R e_{a}$. At fixed ratio $Q / N u_{R B}$ and when $R a$ increases, both ratios $N u_{a} / N u_{R B}$ and $R e_{a} / R e_{R B}$ increase for $Q<0$ and they decrease for $Q>0$. This can easily be explained by the fact that convection is described by a mixture of regimes $I I$ and $I V$ for $\operatorname{Pr}=1$ and the higher $R a$, the lower the portion corresponding to regime $I I$. Besides, $N u_{a} \approx N u_{R B}$ for pure regime $I I$ whereas we have

$$
\frac{N u_{a}}{N u_{R B}}=\left(\frac{R e_{a}}{R e_{R B}}\right)^{3 / 2}=\frac{1}{2}+\frac{1}{2} \sqrt{1-\frac{Q}{N u_{R B}}}
$$

for pure regime $I V$ and $\delta_{u}>\delta_{T}$ (or $\operatorname{Pr} \geqslant 1$ ). To obtain (2.22), the system of Eqs. (2.20) and (2.21) is solved with $c_{1}=c_{3}=0$ (regime $I V$ ), assuming that $N u_{a} \gg 1>\frac{\lambda_{d}}{2} \frac{Q}{N u_{R B}}$ and for $\delta_{u}>\delta_{T}\left[f\left(\delta_{u} / \delta_{T}\right) \approx \delta_{T} / \delta_{u}\right]$. Figures $2(\mathrm{a})$ and $2(\mathrm{~b})$ show that ratios $N u_{a} / N u_{R B}$ and $R e_{a} / R e_{R B}$ tend to the solution given by (2.22) when $R a \rightarrow \infty$ and $\operatorname{Pr}=1$.

Finally, when fixing $R a$ and $Q$, and for $P r \geqslant 1$, ratios $N u_{a} / N u_{R B}$ and $R e_{a} / R e_{R B}$ depend very little on $\operatorname{Pr}$ (see Figs. 2c-d), in agreement with the solution of pure regime $I V_{u}$ (Eq. 2.22). On the contrary, when $\operatorname{Pr}$ decreases from 1 to 0 and even if $R a$ is held constant, both $R e_{R B} \operatorname{Pr}$ and $R e_{a} P r$ decrease. As a result, the importance of regime $I I$ in the mixture of regimes represented in Eq. (2.21) increases and thus ratio $N u_{a} / N u_{R B}$ approaches 1 (Figs. 2c-d).

These results are used in the following section to study the configuration shown in Fig. 1(b) for which both non-dimensional numbers $R a$ and $Q$ are not control parameters. Indeed, they both depend on the temperature of the bulk flow $\left(\bar{T}_{b}\right)$ and thus $\bar{T}_{b}$ need to be either measured or predicted. However, for configuration (b), using definitions (1.1) and (1.12), we have: $R r=R a Q$, with $R r$ the Rayleigh number adopted for IH convection experiment (Eq. 1.2).

\section{Theory for IH convection experiment (configuration b)}

\subsection{Model for the top Nusselt number}

The main assumption of this model is to assume that the flow in the upper half of the configuration shown in Fig. 1(b) is similar to the flow seen in the upper half of the cell of configuration (a). By analogy with $\mathrm{RB}$ convection for which $N u_{R B} \sim R a^{1 / 3}$ means that the two boundary layers behave independently of each other, we assume here that the upper boundary layer is not sensitive to the flow structure that controls the lower boundary layer. Therefore, the thickness of the top BL $\left(\delta_{t o p}\right)$, the heat flux at the top plate $\left(q_{t o p} h\right)$ and the characteristic velocity $\left(U_{b}\right)$ are only controlled by the 
following parameters: $\Delta T=2\left(\bar{T}_{b}-T_{0}\right), q, h$ and $P r$. In non-dimensional form, it is assumed that $N u_{\text {top }}$ and $R e_{b}$ are given by the Nusselt and Reynolds numbers calculated for configuration (a) under the same conditions:

$$
\begin{aligned}
N u_{\text {top }}(R r, \operatorname{Pr}) & =N u_{a}(\operatorname{Ra}, \operatorname{Pr}, Q), \\
\operatorname{Re}_{b}(\operatorname{Rr}, \operatorname{Pr}) & =\operatorname{Re}_{a}(\operatorname{Ra}, \operatorname{Pr}, Q) .
\end{aligned}
$$

Besides, modelling both $N u_{b o t}$ and $N u_{t o p}$ is sufficient to give $R a$ and $Q$ as a function of the two control parameters $R r$ and $P r$. Indeed, using (1.2)-(1.8), (1.1) and (1.12) become

$$
\begin{aligned}
Q(R r, P r) & =N u_{b o t}+N u_{t o p}, \\
R a(R r, P r) & =\frac{R r}{N u_{b o t}+N u_{t o p}} .
\end{aligned}
$$

\subsection{Model for the bottom Nusselt number}

Since the mean temperature gradient is positive throughout the lower boundary layer (see Fig. 1b), forced convection is the main mechanism that drives the heat flux at the lower plate. Assuming static large scale flow, also called 'wind flow', a laminar velocity boundary layer develops against the bottom plate in the same way as a Blasius boundary layer. Using the theory of Prandtl-Blasius-Pohlhausen (Schlichting 1979), the thickness of the thermal boundary layer is given by

$$
\frac{\delta_{T, x}}{x}=\frac{1}{A \mathcal{F}(P r) R e_{x}^{1 / 2}}
$$

with $x$ the horizontal coordinate, $A \approx 0.33$ and $\mathcal{F}(1)=1$. Here, $\delta_{T}$ is defined as $\left(\delta_{T}\right)^{-1}=$ $\left(\frac{\mathrm{d} \Theta_{P}}{\mathrm{~d} z}\right)_{z=0}$, with $\Theta_{P}=\left(T-T_{0}\right) /\left(\bar{T}_{b}-T_{0}\right)$ the non-dimensional difference of temperature. The Prandtl-dependent function $\mathcal{F}$ varies as $\operatorname{Pr}^{1 / 3}$ when $\operatorname{Pr} \geqslant 0.5$, whereas, when $\operatorname{Pr}$ decreases, the exponent of the power-law behaviour of $\mathcal{F}$ increases up to $1 / 2$. In the range $10^{-3} \leqslant \operatorname{Pr} \leqslant 10^{3}, \mathcal{F}$ can be approximated by $\mathcal{F}(\operatorname{Pr})=d_{1} \operatorname{Pr}^{1 / 2} f\left(d_{2} \operatorname{Pr}^{1 / 6}\right)$ with a precision of $\pm 1 \%$, using $f(x)=\left(1+x^{n}\right)^{-1 / n}, n=4, d_{1}=1.68$ and $d_{2}=1.63$.

At the bottom plate $(z=0)$, the local heat flux is the sum of two contributions:

$$
\Phi_{x}=\Phi_{x, P}+\Phi_{x, q} .
$$

$\Phi_{x, P}$ and $\Phi_{x, q}$ represent, respectively, the heat flux given by the theory of Prandtl-BlasiusPohlhausen and the heat flux due to the presence of volumetric heat sources inside the thermal boundary layer:

$$
\begin{aligned}
\Phi_{x, P} & =A \mathcal{F}(P r) R e_{x}^{1 / 2} \frac{\lambda\left(\bar{T}_{b}-T_{0}\right)}{x} \\
\Phi_{x, q} & =B q \delta_{T, x} .
\end{aligned}
$$

In (3.8), $B$ is a numerical constant to be determined using experiments or numerical simulations. In the case of $\Phi_{x, P}=0$ (pure conductive state), $B=0.5$.

Assuming an aspect ratio of 1 for the cell and using (3.7), (3.8) and (3.5), the integration of the local heat flux $\Phi_{x}$ (Eq. 3.6) between $x=0$ and $x=h$ gives

$$
\bar{\Phi}_{b o t}=\frac{1}{h} \int_{0}^{h} \Phi_{x}=\frac{2 A \mathcal{F}(\operatorname{Pr})}{h} \lambda\left(\bar{T}_{b}-T_{0}\right) R e_{b}^{1 / 2}+\frac{2 B q h}{3 A \mathcal{F}(\operatorname{Pr}) \operatorname{Re}_{b}^{1 / 2}} .
$$

Using (3.9) and (1.12), the bottom Nusselt number can be calculated as

$$
N u_{b o t}=\frac{\bar{\Phi}_{b o t} h}{2 \lambda\left(\bar{T}_{b}-T_{0}\right)}=A \mathcal{F}(P r) R e_{b}^{1 / 2}+\frac{2 B}{3 A \mathcal{F}(P r)} \frac{Q}{R e_{b}^{1 / 2}} .
$$


Likewise, from (3.5), the mean bottom boundary layer thickness can be written as:

$$
\frac{\bar{\delta}_{b o t}}{h}=\frac{1}{h^{2}} \int_{0}^{h} \delta_{T, x} \mathrm{~d} x=\frac{2}{3 A \mathcal{F}(\operatorname{Pr}) R_{b}^{1 / 2}} .
$$

\subsection{Mean fluid temperature $\langle\bar{T}\rangle$}

Both models presented in sub-sections 3.1 and 3.2 are based on Blasius profiles for the $z$-evolution of $\bar{T}(z)$ in the two boundary layers. In addition, $\bar{T}(z) \approx \bar{T}_{b}$ in the bulk flow i.e. for $\bar{\delta}_{b o t} \ll z \ll h-\delta_{\text {top }}$. Thus, for configuration (b), using $(2.12 b), \bar{T}(z)$ can be expressed as

$$
\frac{\bar{T}_{b}-\bar{T}(z)}{\bar{T}_{b}-T_{0}}= \begin{cases}1-\Theta_{P}\left(\frac{z}{\bar{\delta}_{b o t}}\right), & \text { for } z \leqslant h / 2, \\ 1-\Theta_{P}\left(\frac{h-z}{\bar{\delta}_{t o p}}\right), & \text { for } h / 2 \leqslant z \leqslant h .\end{cases}
$$

The thicknesses of the bottom and top boundary layers $\left(\bar{\delta}_{b o t}\right.$ and $\left.\bar{\delta}_{t o p}\right)$ are given by (2.1) and (3.11), respectively. Using (3.12), the mean fluid temperature can be calculated yielding to

$$
\begin{aligned}
\frac{\bar{T}_{b}-\langle\bar{T}\rangle}{\bar{T}_{b}-T_{0}} & =\lambda_{d} \frac{\bar{\delta}_{b o t}+\bar{\delta}_{t o p}}{h}, \\
& =\lambda_{d}\left(\frac{2}{3 A \mathcal{F}(P r) R e_{b}^{1 / 2}}+\frac{1}{2 N u_{R B}}\right),
\end{aligned}
$$

with $R e_{b}(R r, P r)$ (Eq. 3.2) and $N u_{R B}(R a, P r)$ with $R a(R r, P r)$ (Eq. 3.4).

\section{Comparison between theories and numerical results}

Theoretical predictions for $N u_{t o p}, N u_{\text {bot }}$ and mean fluid temperature are tested below using experimental and numerical results on three-dimensional IH convection (see Table 1 and Goluskin (2015) for a literature survey). A comparison between the theories and twodimensional simulations of IH convection (Goluskin \& Spiegel 2012; Goluskin \& van der Poel 2016; Wang et al. 2021) is given in Appendix A.

\subsection{Top Nusselt number}

Figures 3(a) and 3(b) show the variations of $N u_{t o p}$ (filled squares) defined by (1.8) and given by Goluskin \& van der Poel (2016) for IH convection simulations. The variations of the Nusselt number obtained for standard RB simulations are also shown (data from Pandey \& Verma (2016) and Shishkina et al. (2017), open symbols). Clearly, at constant $R a$ and $\operatorname{Pr}$ numbers, $N u_{\text {top }}$ is slightly lower than $N u_{R B}$. Dashed and solid lines represent, respectively, GL theory for RB convection (Eqs. 2.18 and 2.19) and for RB convection with volumetric energy sources (Eqs. 2.20 and 2.21). For the latter case, $Q$ and $R a$ are calculated using (3.3), (3.4) and the data of Goluskin \& van der Poel (2016). A good agreement between the numerical simulations and the extension of GL theory is observed both by plotting $N u_{t o p}$ as a function of $R a$ for $\operatorname{Pr}=1$ (Fig. 3a) and by considering the variations of $N u_{\text {top }}$ with $\operatorname{Pr}$ for $R a \approx 1.7 \times 10^{6}$ (Fig. $3 \mathrm{~b}$ ). 

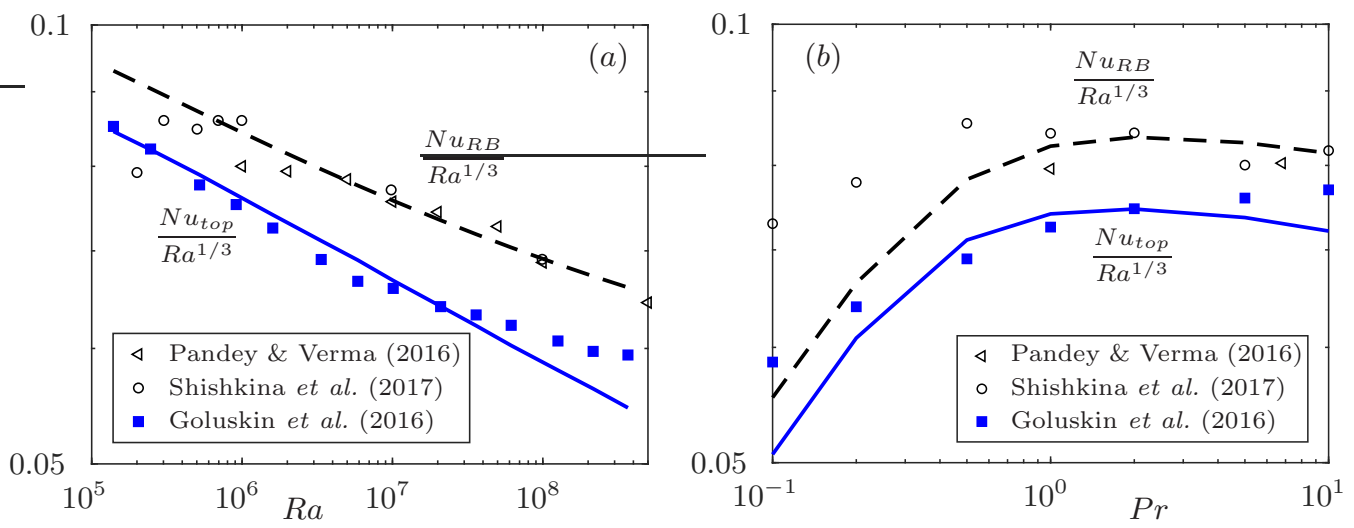

FiguRE 3 . The compensated Nusselt number for standard RB convection (open symbols) and for IH convection (filled squares) as a function of $R a$ for $\operatorname{Pr}=1$ (a) and against $\operatorname{Pr}$ for $R a \approx 1.7 \times 10^{6}$ (b). For IH convection, $R a$ numbers are calculated using (3.4). Dashed lines: Grossmann \& Lohse (2001) theory with prefactors given by Stevens et al. (2013) (Eqs. 2.18 and 2.19). Solid blue lines: extension of GL theory for convection with volumetric energy sources (Eqs. 2.20 and 2.21).
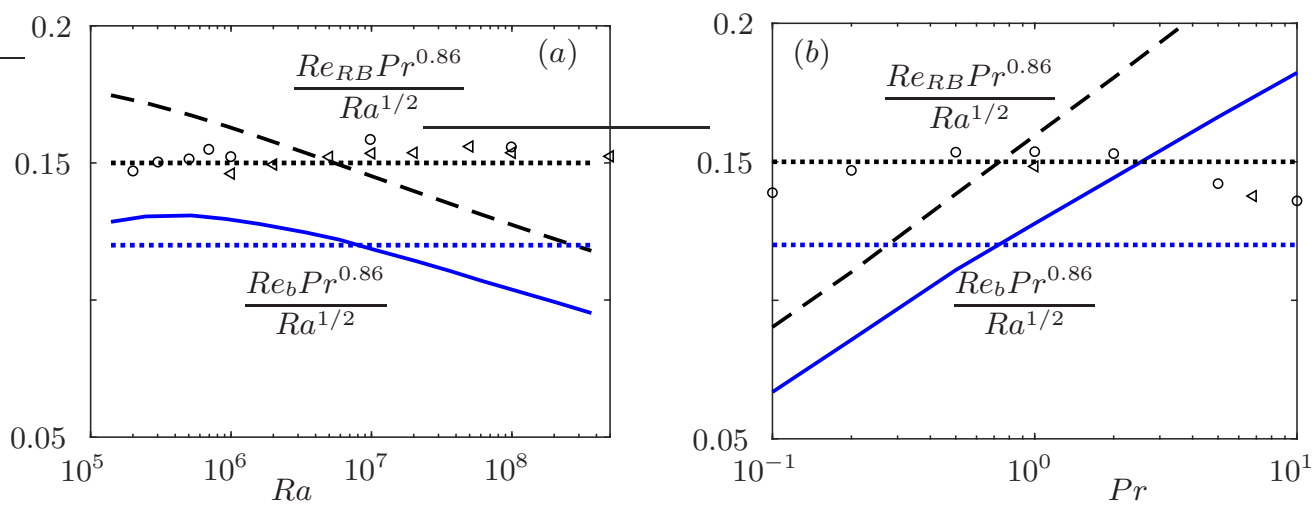

FiguRE 4. The compensated Reynolds number for standard RB convection as a function of $R a$ for $\operatorname{Pr}=1$ (a) and against $\operatorname{Pr}$ for $R a \approx 1.7 \times 10^{6}$ (b) (symbols as Fig. 3). Also shown: the predictions of the GL theory (dashed lines, Eqs. 2.18 and 2.19) and those of its extension to convection with volumetric heat source (solid lines, Eqs. 2.20 and 2.21). For the second case, $R a$ and $Q$ are calculated using (3.4) and (3.3) and the data of Goluskin \& van der Poel (2016). The horizontal lines represent power-law behaviours (upper line: (4.1), lower line: (4.2)).

\subsection{Bottom Nusselt number}

The theory developed in sub-section 3.2 to predict the variations of $N u_{b o t}$ is based on forced convection in the lower half of the cell and the theory of Blasius. Therefore, to get $N u_{\text {bot }}$ with good precision, a very good estimate of the Reynolds number is necessary (see Eq. 3.10). However, to date there are no experimental or numerical measurements for either $\operatorname{Re}_{b}(R r, P r)$ or $\operatorname{Re}_{a}(R a, P r, Q)$ in 3D. Even for RB convection, experimental and numerical measurements of Reynolds number have larger uncertainties than those of Nusselt number because they require measurement of velocity fluctuations throughout the cell. In addition, several definitions of $R e$ are given in the literature (root mean square of the velocity, root mean square of the vertical velocity, maximum of the velocity fluctuations, maximum mean velocity along the heated plate). Likewise, the GL theory is less effective in predicting $R e_{R B}$ than $N u_{R B}$. Figures $4(\mathrm{a})$ and $4(\mathrm{~b})$ show the variations 

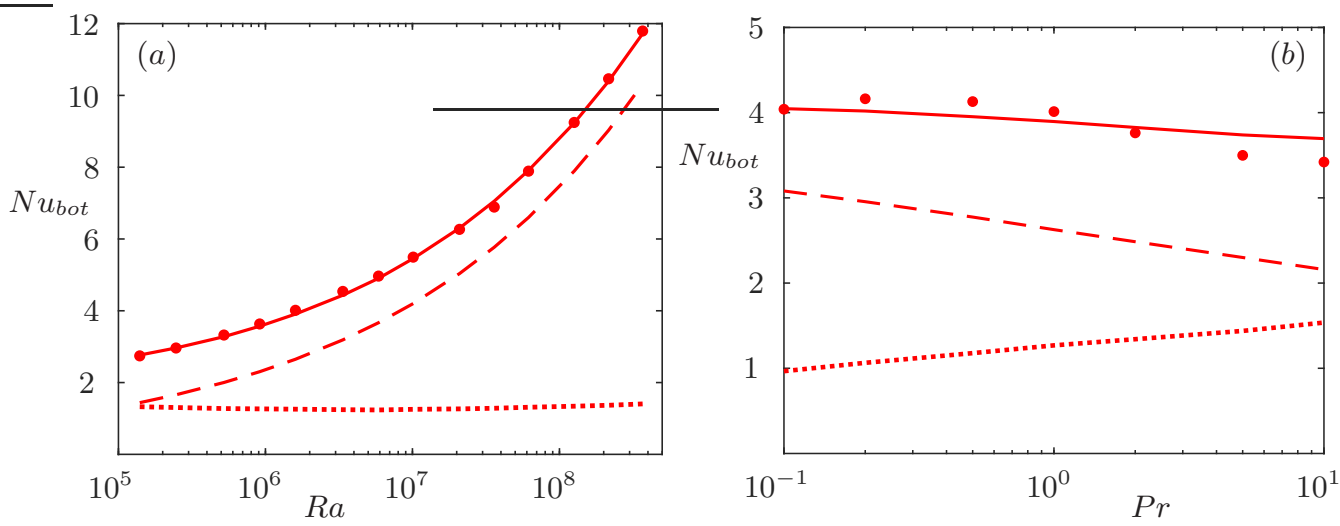

Figure 5. Nu bot versus $R a$ (a) and $\operatorname{Pr}$ (b). Symbols: data from Goluskin \& van der Poel (2016). Solid lines: (3.10) with $A=0.21$ and $B=0.4$. Dashed lines: first term of (3.10) $\left(\propto \sqrt{R e} \propto R a^{1 / 4}\right)$. Dotted lines: second term of $(3.10)(\propto Q / \sqrt{R e})$.

of $R e_{R B}$ as a function of $R a$ and $P r$ given by Pandey \& Verma (2016) and Shishkina et al. (2017). For both cases i.e. $\operatorname{Pr}=1,10^{5} \leqslant R a \leqslant 5 \times 10^{8}$ (Fig. $4 \mathrm{a}$ ) and $R a=1.7 \times 10^{6}$, $0.1 \leqslant P r \leqslant 10, R e_{R B}$ can be fitted by a power-law of $R a$ and $\operatorname{Pr}$ as

$$
R e_{R B} \approx 0.15 \operatorname{Ra}^{1 / 2} \mathrm{Pr}^{-\gamma},
$$

with $\gamma \approx 0.86$. As no data on $R e_{a}$ is available, the same power-law behaviour will be used for $R e_{a}$. In addition, the extension of the GL theory (see section 2) predicts that $R e_{a} / R e_{R B} \approx 0.8$ in the range of $R a, P r$ and $Q$ investigated by Goluskin \& van der Poel (2016) (see Figs. 4a,b). Thus, the following equation will be use to give $R e_{a}$ or $R e_{b}$ as a function of $R a$ and $P r$ :

$$
\operatorname{Re}_{b}(R, \operatorname{Pr})=\operatorname{Re}_{a}(\operatorname{Ra}, \operatorname{Pr}, Q)=0.12 \operatorname{Ra}^{1 / 2} \operatorname{Pr}^{-\gamma} .
$$

Using (4.2), (3.10) gives $N u_{b o t}$ as a function of $R a, P r$ and $Q$. Note that $R a$ and $Q$ are calculated from the data of Goluskin \& van der Poel (2016) and using (3.3) and (3.4). Figures 5 (a-b) show that the equation (3.10) describes well both the evolution of $N u_{\text {bot }}$ with $R a$ and with $\operatorname{Pr}$, using $A=0.21$ (not far away from 0.33 ) and $B=0.4$ (close to the value 0.5 which characterizes a purely conductive boundary layer).

\subsection{Mean fluid temperature $\langle\bar{T}\rangle$}

The model presented in section 3 also predicts the evolution with $R a$ and $\operatorname{Pr}$ of the difference between the mean fluid temperature $(\langle\bar{T}\rangle)$ and the temperature of the bulk flow $\left(\bar{T}_{b}\right)$. Figures 6 (a-b) show a good agreement between (3.14) and the numerical results of Goluskin (2015) using no new adjustable parameters. Indeed, as for figures 5 (a-b), $A$ is taken equal to 0.21 whereas the displacement thickness of the mean temperature profile is given by the Blasius theory $\left(\lambda_{d}=0.57\right)$. As shown by $(3.13)$, the dimensionless temperature difference $\left(\bar{T}_{b}-\langle\bar{T}\rangle\right) /\left(\bar{T}_{b}-T_{0}\right)$ is equal to the sum of the thicknesses of the two boundary layers. For $R a>10^{5}$, as $\bar{\delta}_{b o t} \gg \bar{\delta}_{\text {top }}$ and $R e_{b} \propto R a^{1 / 2}$ (see Eq. (4.2)), (3.14) can be approximated by

$$
\frac{\bar{T}_{b}-\langle\bar{T}\rangle}{\bar{T}_{b}-T_{0}} \approx \lambda_{d} \frac{\bar{\delta}_{b o t}}{h} \propto R a^{-1 / 4}
$$

$\frac{\bar{\delta}_{b o t}}{h} \propto R e_{b}{ }^{-1 / 2}$ (see (3.11)) and $R e_{b} \propto R a^{1 / 2}$ (4.2), As Rayleigh number increases, 

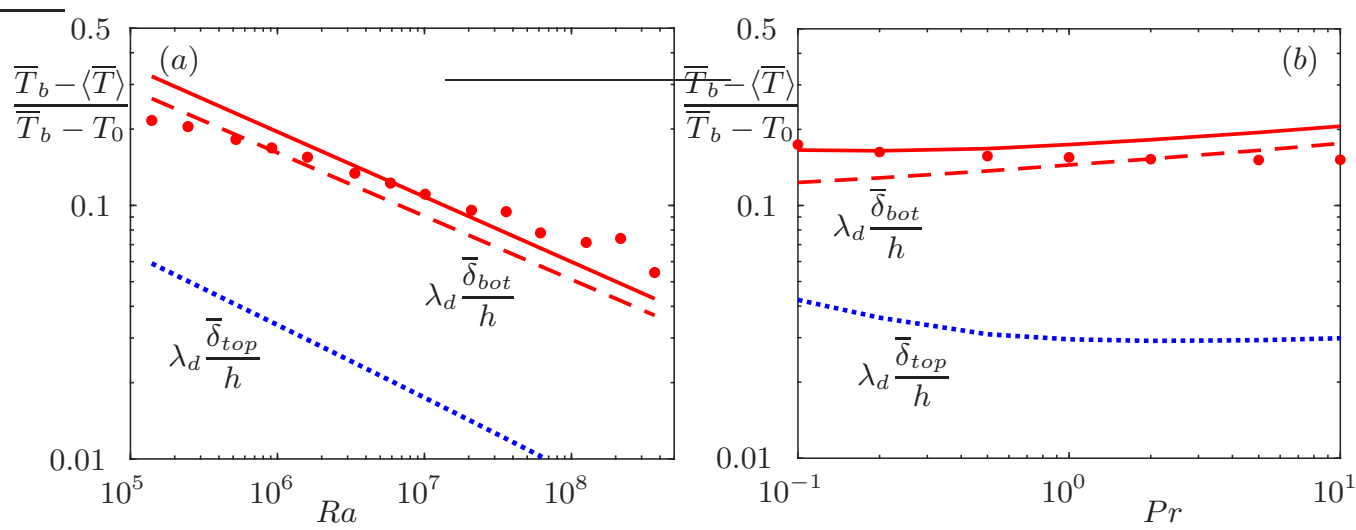

FiguRE 6. Difference between the temperature of the bulk flow $\left(\bar{T}_{b}\right)$ and the mean fluid temperature $(\langle\bar{T}\rangle)$ as a function of $R a$ (a) and $\operatorname{Pr}$ (b). Symbols: data from Goluskin \& van der Poel (2016). Solid lines: (3.14) with $A=0.21$ and $\lambda_{d}=0.57$. Dashed lines: first term of (3.14) i.e. $\lambda_{d} \frac{\bar{\delta}_{b o t}}{h} \propto R e_{b}{ }^{-1 / 2} \propto R a^{-1 / 4}$. Dotted blue lines: second term of (3.14) i.e. $\lambda_{d} \frac{\bar{\delta}_{t o p}}{h} \propto 1 / N u_{R B} \propto R a^{-\theta_{R B}}$ with $\theta_{R B} \approx 0.3$ (see sub-section 4.4).

the thicknesses of the upper and lower boundary layers decrease which also results in a decrease of $\bar{T}_{b}-\langle\bar{T}\rangle$.

\subsection{Approximation of $N u_{\text {top }}$ and $N u_{\text {bot }}$ by power-laws of $R r$}

For $10^{5} \leqslant R a \leqslant 10^{9}$ and $\operatorname{Pr} \geqslant 1$, numerical simulations (Pandey \& Verma 2016; Shishkina et al. 2017; Goluskin \& van der Poel 2016) and GL theory show that both $N u_{R B}$ and $N u_{\text {top }}$ can be approximated by a power-law of $R a$ with an exponent $\theta_{R B} \approx 0.3$ (see also Fig. 3a). By neglecting $N u_{\text {bot }}$ in front of $N u_{t o p},(3.4)$ yields to $R a \sim R r^{1 /\left(1+\theta_{R B}\right)}$. Consequently, we get:

$$
N u_{\text {top }} \approx 0.18 R r^{\frac{\theta_{R B}}{\left(1+\theta_{R B}\right)}} \approx 0.18 R r^{0.23}
$$

This scaling for $N u_{t o p}$ is consistent with the independent theoretical findings of Wang et al. (2021). Indeed, for regimes $I_{\infty}^{<}$and $I V_{u}$, Wang et al. (2021) have shown that $Q \approx \tilde{\Delta}^{-1} \sim R r^{1 / 4}$ whereas for regimes $I_{u}$ and $I_{l}, Q \approx \tilde{\Delta}^{-1} \sim R r^{1 / 5}$.

Using (4.2) and assuming again $Q \approx N u_{\text {top }},(3.10)$ becomes

$$
\begin{aligned}
N u_{b o t} & \approx 0.11 \operatorname{Pr}^{-\Gamma} \operatorname{Rr}^{\frac{1}{4\left(1+\theta_{R B}\right)}}+0.42 \operatorname{Pr}^{\Gamma} \operatorname{Rr}^{\frac{\theta_{R B}-1 / 4}{1+\theta} R B}, \\
& \approx 0.11 \mathrm{Pr}^{-0.10} \mathrm{Rr}^{0.19}+0.42 \operatorname{Pr}^{0.10} \mathrm{Rr}^{0.04} .
\end{aligned}
$$

Indeed, for $\operatorname{Pr} \geqslant 1, \mathcal{F}(P r) \approx P r^{1 / 3}$ and $\Gamma=\frac{\gamma}{2}-\frac{1}{3} \approx 0.10$. Equation (4.6) shows that $N u_{\text {bot }}$ can be expressed by the sum of two power-laws of $R r$ with exponents 0.04 and 0.19 (see also Fig. 7). This explains the high variability of the measured exponents when the experimental or numerical results are fitted by a single power law (see Table 1$)$. Since the experiments of Ralph et al. (1977) were performed for higher $R r$ numbers $\left(4 \cdot 10^{8}-10^{12}\right)$ than the other works, the corresponding exponent $(0.17)$ is larger than the exponents calculated using the other results $(\sim 0.10)$. Of course, the observed differences are also due to experimental uncertainties, boundary conditions and the way the thermal power $q_{v}$ is imposed in volume in the fluid. 


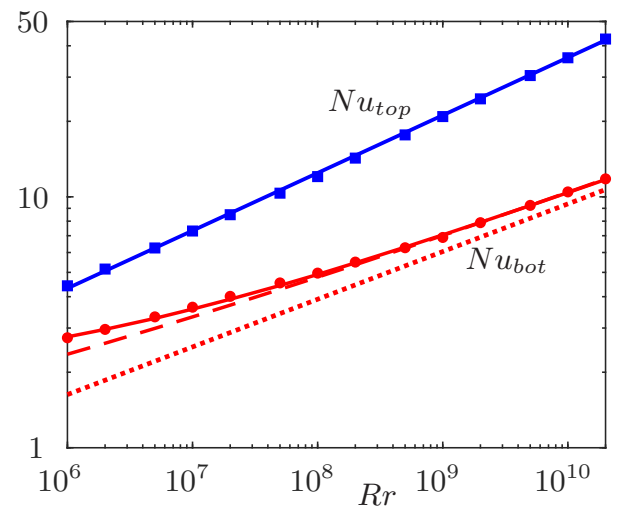

Figure 7. $N u_{\text {top }}$ (squares) and $N u_{\text {bot }}$ (circles) versus $R r$ for $\operatorname{Pr}=1$ (data from Goluskin \& van der Poel (2016)). Solid upper line: $N u_{t o p} \propto R r^{0.23}$ (Eq. 4.4). Solid lower line: (3.10) with $A=0.21$ and $B=0.4$. Dashed line: $N u_{b o t}=0.11 R r^{0.19}+0.42 R r^{0.04}$ (Eq. 4.6). Dotted line: $N u_{\text {bot }}=0.11 R r^{0.19}$.

\section{Conclusions}

Grossmann \& Lohse (2001) theory has been extended here to RB convection with volumetric energy sources in the fluid (configuration a). The two equations of the GL theory (2.18)-(2.19) have been modified to take account the effect of the new non-dimensional parameter $Q=q h^{2} /(\lambda \Delta T)$. Equations (2.20) and (2.21) predict the evolution of $N u_{a}$ and $R e_{a}$ as a function of $R a, \operatorname{Pr}$ and $Q$. Besides, these two equations can also describe the top Nusselt number $N u_{t o p}$ and the Reynolds number of an IH convection experiment (configuration b). In this case, the non-dimensional parameter $R r=R a Q$ is the control parameter of the IH convection system whereas $R a$ and $Q$ are theoretically modelled using $Q=N u_{\text {top }}+N u_{\text {bot }}$, with $N u_{\text {top }}=N u_{a}(R a, \operatorname{Pr}, Q)$ and $N u_{b o t}\left(\operatorname{Re}_{a}, \operatorname{Pr}, Q\right)$. The bottom Nusselt number is the sum of two terms (Eq. 3.10). The first term can be modelled using the Prandtl-Blasius-Pohlhausen theory and is proportional to square root of the Reynolds number. The second term comes from the presence of volumetric energy sources and is proportional to $Q / \sqrt{R e}$. In agreement with the independent theoretical findings of Wang et al. (2021), for high $R r$ numbers, $N u_{t o p}$ scales as $R r^{\left.\frac{\theta_{R B}}{(1+\theta} R B\right)}$, with $\theta_{R B}$ the exponent of the power-law behaviour of $N u_{R B}$ with $R a$. These predictions are confirmed by experimental and numerical results and show that natural and forced convections are the two mechanisms that control the heat fluxes in an IH convection experiment.

\section{Acknowledgements}

Bernard Castaing is gratefully thanked for his suggestions and the review of the article.

The author reports no conflict of interest.

\section{Appendix A. Comparison between theories and numerical results for two-dimensional IH convection}

For two-dimensional IH convection, the predictions of the theoretical work presented in section 3 can be tested thanks to the numerical investigations of Goluskin \& Spiegel (2012); Wang et al. (2021). Figures 8(a-b) show the variations of $N u_{t o p}$ and $R e$ as a function of the Rayleigh number $\left(R a=\operatorname{Rr} /\left(N u_{t o p}+N u_{b o t}\right)\right)$, for $\operatorname{Pr}=1$. For 

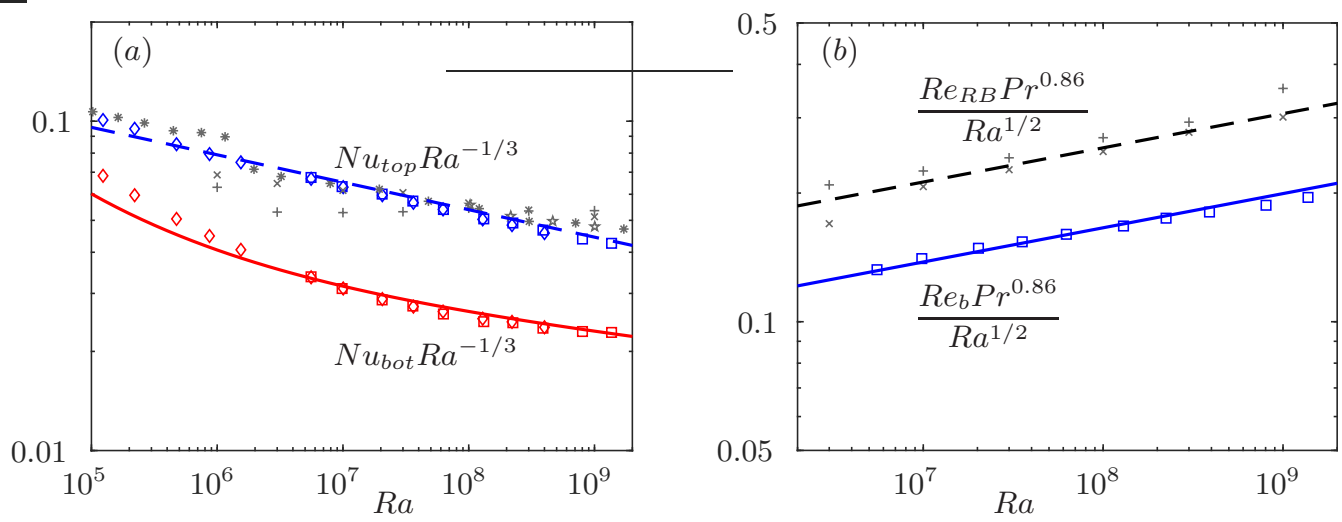

Figure 8. Results for two-dimensional IH and RB convection. (a) $N u_{\text {top }}$ and $N u_{\text {bot }}$ compensated by $R a^{1 / 3}$ as a function of $R a$. Data from Goluskin \& Spiegel (2012) (diamonds) and Wang et al. (2021) (squares), $P r=1$. The results for RB convection $\left(N u_{R B} R a^{-1 / 3}\right)$ are also shown: Johnston \& Doering (2009) (asterisks), Zhu et al. (2018) (stars) and Zhang et al. (2017) (plusses: $\operatorname{Pr}=0.7$, crosses: $P r=5.3$ ). Blue dashed line: (A 1). Red solid line: (3.10) with $A_{2 D}=0.28$ and $B_{2 D}=0.4$. (b) Compensated Reynolds number $\left(\operatorname{RePr}^{0.86} / R a^{1 / 2}\right)$ vs. $R a$. Blue solid line: (A 2 ).

comparison purposes, the measured Nusselt and Reynolds numbers for RB convection are also displayed. We observe very little difference between $N u_{\text {top }}$ and $N u_{R B}$ whereas the Reynolds number is about $65 \%$ lower for IH convection than for RB convection. With good precision, $N u_{t o p}$ and $R e_{b}$ can be approximated by power laws of $R a$ as:

$$
\begin{aligned}
N u_{\text {top }} & \approx N u_{R B} \approx 0.25 R a^{0.25}, \\
R e_{b} & \approx 0.65 R e_{R B} \approx 0.038 \operatorname{Pr}^{0.86} R^{0.58} .
\end{aligned}
$$

The results for $2 \mathrm{D}$ IH convection are therefore slightly different from those obtained for 3D IH convection. In 3D, numerical data of Goluskin (2015) and extension of the GL theory show that $0.83 \leqslant N u_{t o p} / N u_{R B} \leqslant 0.91$ and $R e_{b} \approx 0.8 R e_{R B}$ (using GL theory) whereas $N u_{\text {top }} \approx N u_{R B}$ and $R e_{b} \approx 0.65 R e_{R B}$ in 2D. However, beyond these small differences, the $2 \mathrm{D}$ and $3 \mathrm{D}$ numerical results validate the theoretical approach chosen here to separate the IH convection cell into two distinct parts. The upper half of the cell behaves like the upper half of a RB convection cell $\left(N u_{t o p} \sim N u_{R B}\right)$ and controls the Reynolds number. The heat transfer at the bottom plate is controlled by the Reynolds number and using the theory of Blasius, $N u_{b o t}$ is given by (3.10). Indeed, Figure 8(a) shows a good agreement between results of Wang et al. (2021) (red squares) and (3.10) (red solid line) using $A_{2 D}=0.28$ and $B_{2 D}=B_{3 D}=0.4$. In $2 \mathrm{D}$, the parameter $A$ is therefore slightly higher than in $3 \mathrm{D}\left(A_{3 D} \approx 0.22\right)$ and closer to the value for a Blasius boundary layer $(A \approx 0.33)$. A greater difference is observed between the data from Goluskin \& Spiegel (2012) (red diamonds) and (3.10). This is probably due to the fact that the equation that gives the Reynolds number (A 2) is less well verified when the Rayleigh number is low.

The updown asymmetry that turbulent convection induces is quantified measuring the coefficient $\alpha$. From definitions given by (1.5)-(1.7), $\alpha$ is directly linked to the ratio of $N u_{\text {top }}$ over $N u_{\text {bot }}$

$$
2 \alpha=\frac{\frac{N u_{t o p}}{N u_{b o t}}-1}{\frac{N u_{t o p}}{N u_{b o t}}+1} .
$$




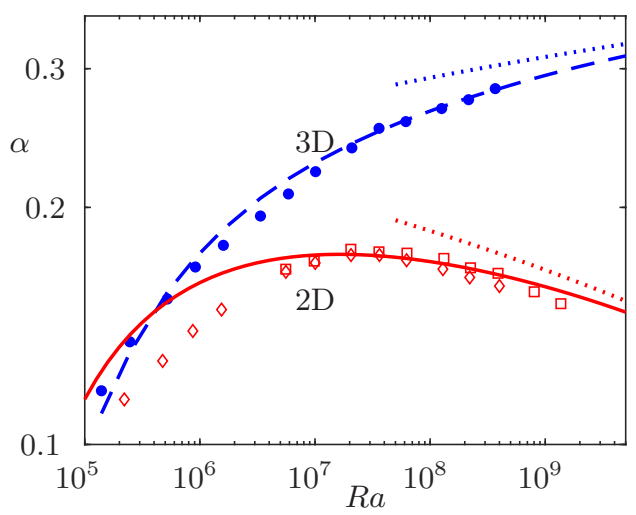

Figure 9. Asymmetry coefficient $\alpha$ versus $R a$ for 2D (red open symbols) and 3D IH convection (blue circles: data of Goluskin \& van der Poel (2016)). Red solid line: (A 3) and using (A 1), (A 2) and (3.10). Blue dashed line: (A 3) and using (4.4), (4.2) and (3.10). Lower and upper dotted lines: (A 3) with $N u_{\text {top }} / N u_{\text {bot }} \sim R a^{-0.04}$ and $N u_{\text {top }} / N u_{\text {bot }} \sim R a^{0.05}$, respectively.

Both for 2D and 3D IH convection, Figure 9 shows that the theory presented in section 3 describes well the evolution of the asymmetry coefficient with $R a$. However, the evolution of $\alpha$ with $R a$ is different in $2 \mathrm{D}$ than in 3D. In particular, for $2 \mathrm{D}, \alpha$ saturates and even decreases when $R a$ increases. This behaviour is well described by (3.10). For high $R a$ numbers, (3.10) can be approximated by $N u_{\text {bot }} \sim R e^{1 / 2}$. Using (A 2) (2D) or (4.2) (3D), we obtain $N u_{\text {bot }} \sim R a^{0.29}$ for $2 \mathrm{D}$ while $N u_{\text {bot }} \sim R a^{0.25}$ for $3 \mathrm{D}$. As $N u_{\text {top }} \sim R a^{0.25}$ for 2D (A 1) and $N u_{\text {top }} \sim R a^{0.3}$ for 3D (see subsection 4.4 ), $N u_{\text {top }} / N u_{\text {bot }}$ varies as $R a^{-0.04}$ for $2 \mathrm{D}$ and as $R a^{0.05}$ for $3 \mathrm{D} \mathrm{IH}$ convection. That is why, for $R a \gtrsim 10^{7}, \alpha$ decreases with $R a$ in $2 \mathrm{D}$ while $\alpha$ continues to increase with $R a$ in $3 \mathrm{D}$ (see Fig. 9).

\section{REFERENCES}

Boulllaut, V., Lepot, S., Aumaître, S. \& Gallet, B. 2019 Transition to the ultimate regime in a radiatively driven convection experiment. J. Fluid Mech. 861, R5.

Creyssels, M. 2020 Model for classical and ultimate regimes of radiatively driven turbulent convection. J. Fluid Mech 900 (A39).

Goluskin, D. 2015 Internally Heated Convection and Rayleigh-Bénard Convection. Springer.

Goluskin, D. \& van Der Poel, E. P. 2016 Penetrative internally heated convection in two and three dimensions. J. Fluid Mech. 791, R6.

Goluskin, D. \& Spiegel, E. A. 2012 Convection driven by internal heating. Phys. Lett. A 377, 83-92.

Grossmann, S. \& Lohse, D. 2000 Scaling in thermal convection: A unifying view. J. Fluid Mech. 407, 27-56.

Grossmann, S. \& Lohse, D. 2001 Thermal convection for large Prandtl numbers. Phys. Rev. Lett. 86, 3316-3319.

Jahn, M. \& Reineke, H. H. 1974 Free convection heat transfer with internal heat sources, calculations and measurements. In Proc. 5th Int. Heat Transfer Conf., pp. 74-78.

Johnston, H. \& Doering, C. R. 2009 Comparison of turbulent thermal convection between conditions of constant temperature and constant flux. Phys. Rev. Lett. 102, 064501.

Kulacki, F. A. \& Goldstein, R. J. 1972 Thermal convection in a horizontal fluid layer with uniform volumetric energy sources. J. Fluid Mech. 55 (2), 271-287.

LeE, S. D., LeE, J. K. \& Suh, K. Y. 2007 Boundary condition dependent natural convection in a rectangular pool with internal heat sources. Trans. ASME J. Heat Transfer 129 (5), 679-682. 
Lepot, S., Aumaître, S. \& Gallet, B. 2018 Radiative heating achieves the ultimate regime of thermal convection. Proc. Natl Acad. Sci. USA 115, 8937-8941.

Mayinger, F., Jahn, M., Reineke, H. H. \& Steinberner, U. 1975 Examination of thermohydraulic processes and heat transfer in a core melt. Tech. Rep.. Hannover Technical University, Hannover, Germany.

Pandey, A. \& Verma, M. K. 2016 Scaling of large-scale quantities in Rayleigh-Bénard convection. Phys. Fluids 28 (9), 095105.

Ralph, J. C., McGreevy, R. \& Peckover, R. S. 1977 Experiments in turbulent thermal convection driven by internal heat sources. In Heat Transfer and Turbulent Buoyant Convection: Studies and Applications for Natural Environment, Buildings, Engineering Systems, pp. 587-599.

Roberts, P. H. 1967 Convection in horizontal layers with internal heat generation. Theory. $J$. Fluid Mech. 30 (1), 33-49.

Schlichting, H. 1979 Boundary-Layer Theory, 7th edn. New York: McGraw Hill.

Shishkina, O., Emran, M. S., Grossmann, S. \& Lohse, D. 2017 Scaling relations in largePrandtl-number natural thermal convection. Phys. Rev. Fluids 2, 103502.

Stevens, R. J. A. M., van der Poel, E. P., Grossmann, S. \& Lohse, D. 2013 The unifying theory of scaling in thermal convection: the updated prefactors. J. Fluid Mech. 730, 295308.

Wang, Q., Shishkina, O. \& Lohse, D. 2021 Scaling in internally heated convection: A unifying theory. Geophys. Res. Lett. 48 (4), e2020GL091198.

Wörner, M., Schmidt, M. \& Grötzbach, G. 1997 Direct numerical simulation of turbulence in an internally heated convective fluid layer and implications for statistical modeling. $J$. Hydraul. Res. 35 (6), 773-797.

Zhang, Y., Zhou, Q. \& Sun, C. 2017 Statistics of kinetic and thermal energy dissipation rates in two-dimensional turbulent Rayleigh-Bénard convection. J. Fluid Mech. 814, 165-184.

Zhu, X., Mathai, V., Stevens, R. J. A. M., Verzicco, R. \& Lohse, D. 2018 Transition to the ultimate regime in two-dimensional Rayleigh-Bénard convection. Phys. Rev. Lett. 120, 144502 . 